\title{
Preoperative Surgical Risk Assessment by Evaluation of Tumor Blood Flow Using Perfusion Imaging by Computed Tomography in Transsphenoidal Surgery for Pituitary Adenoma
}

Nakamasa Hayashi ( $\nabla$ n.hayashi@scchr.jp )

Shizuoka Cancer Center Hospital https://orcid.org/0000-0003-4170-7304

Koichi Mitsuya

Shizuoka Cancer Center Hospital

Shoichi Deguchi

Shizuoka Cancer Center Hospital

Atsushi Urikura

Shizuoka Cancer Center Hospital

Masahiro Endo

Shizuoka Cancer Center Hospital

\section{Research article}

Keywords: CT, Perfusion study, Pituitary adenoma, Postoperative complication, Suprasellar extension, Transsphenoidal surgery

Posted Date: July 9th, 2020

DOI: https://doi.org/10.21203/rs.3.rs-39578/v1

License: (c) (i) This work is licensed under a Creative Commons Attribution 4.0 International License.

Read Full License 


\section{Abstract}

Background: Complete removal of large and giant pituitary adenomas (PAs) with suprasellar extension by transsphenoidal surgery (TSS) is sometimes difficult. Swelling and bleeding of a residual suprasellar tumor after incomplete removal via TSS can compress the surrounding neurovascular structures, resulting in postoperative complications. We measured PA blood flow by perfusion computed tomography (PCT) and assessed its usefulness in the preoperative prediction of postoperative hemorrhagic complications after TSS.

Method: We performed PCT in 28 patients with PAs with suprasellar extension before TSS. Perfusion studies were performed with a 320-row multidetector computed tomography system. We measured cerebral blood volume (CBV) and cerebral blood flow (CBF) of the tumor (CBVt and CBFt) and normalappearing white matter (CBVw and CBFw).

Results: In all patients, CBVt was greater than CBVw. There was a statistically significant difference between CBFt and CBFw $(p<0.0001)$. The patients were divided into 2 groups according to CBFt: high $\mathrm{CBF}$ (CBFt> mean value) and low CBF (CBFt< mean value). There were no statistically significant differences between the 2 groups in terms of gender, age, maximum tumor diameter, suprasellar extension grade, and extent of resection. Two of the 7 patients in the high CBF group suffered from visual acuity deterioration and visual field stenosis due to postoperative hemorrhage, while no patients in the low CBF group did $(p=0.014)$.

Conclusions: PCT may be useful in the preoperative prediction of postoperative residual tumor hemorrhage, which can be a hazardous postoperative complication after TSS for large and giant PAs.

\section{Introduction}

Transsphenoidal surgery (TSS) is often the first choice for the treatment of most pituitary adenomas, and the clinical outcomes are satisfactory when using either an endoscope or a microscope $[4,10,11,16]$. However, the surgical treatment of large and giant pituitary adenomas with suprasellar extension has been controversial. Some tumor characteristics such as a dumbbell-shaped adenoma, a multi-lobular adenoma, asymmetrical suprasellar extension, and a normal-sized pituitary fossa with a predominant suprasellar portion are not suitable indications for TSS [6]. Insufficient transsphenoidal resection puts patients at risk of postoperative bleeding, edema, and increased mass effect of the residual tumor.

Perfusion imaging by computed tomography (PCT) reflects blood flow and capillary condition, making it valuable in the assessment of brain tumors. PCT has been reported to be useful in differentiating malignant brain tumors, grading gliomas, and predicting prognosis after treatment $[2,15,19]$. Reflecting their neovascularization and increased angiogenic activity, high-grade gliomas have greater cerebral blood volume (CBV) and cerebral blood flow (CBF) compared with low-grade gliomas [2, 19]. We measured the $\mathrm{CBV}$ and $\mathrm{CBF}$ of pituitary tumors to assess tumor vascularization and evaluated the 
association of elevated CBV and CBF with the incidence of postoperative hemorrhage after TSS for pituitary adenoma.

\section{Methods \\ Patients}

This retrospective study was approved by the institutional review board of the Shizuoka Cancer Center, and the requirement for informed consent was waived. All methods were performed in accordance with relevant guidelines and regulations.

We included 28 patients with pituitary adenomas treated by TSS at the Shizuoka Cancer Center from March 2015 to December 2019. There were 12 men and 16 women between 29 and 84 years of age (mean age: 56.8 years).

\section{Image Acquisition}

PCT was performed before surgery using a 320-row multidetector computed tomography system (Aquillion ONE; Canon Medical Systems, Tochigi, Japan). Seven seconds after intravenously injecting $50 \mathrm{~mL}$ ( $5 \mathrm{~mL} / \mathrm{s}$ ) of a nonionic contrast medium (Iomeron, $350 \mathrm{mg} / \mathrm{mL}$; Bracco-Eisai, Tokyo, Japan) using a power injector, dynamic scanning was started, and tissue attenuation of the contrast medium was monitored.

Acquired data were analyzed using the delay-insensitive singular value decomposition plus perfusion algorithm on the computed tomography scanner console. The CBV, CBF, mean transit time, and time to peak were displayed automatically. Regions of interest were placed manually at the tumor and normalappearing white matter.

A total of 23 dynamic scans were performed for each patient, and radiation doses for PCT were $157.5 \mathrm{mGy}$ per the volume computed tomography dose index and $2520 \mathrm{mGy} \square \mathrm{cm}$ per the dose length product.

\section{Statistical Analysis}

Analyses were performed using version 11 of the JMP® software (SAS Institute Inc., Tokyo, Japan). The differences in $\mathrm{CBV}$ and $\mathrm{CBF}$ between the tumor (CBVt and CBFt) and normal-appearing white matter ( $\mathrm{CBVw}$ and $\mathrm{CBFw}$ ) were compared by the Wilcoxon rank sum test. Clinical characteristics were compared using the chi-square test. $P<0.05$ was considered statistically significant.

\section{Results}

CBVt ranged from 3.0 to $8.7(5.1 \pm 1.3) \mathrm{mL} / 100 \mathrm{~g}$, and $\mathrm{CBVw}$ ranged from 1.5 to $2.7(2.0 \pm 0.3) \mathrm{mL} / 100 \mathrm{~g}$. In all patients, $\mathrm{CBVt}$ was greater than $\mathrm{CBVw}$. There was a statistically significant difference between $\mathrm{CBV} t$ and $\mathrm{CBVw}_{w}(\mathrm{p}<0.0001$; Fig. 1A). 
CBFt ranged from 28.4 to $133.2(51.3 \pm 27.7) \mathrm{mL} / \mathrm{min} / 100 \mathrm{~g}$, and CBFw ranged from 18.4 to $31.3(24.1 \pm$ 3.6) $\mathrm{mL} / \mathrm{min} / 100 \mathrm{~g}$. There was a statistically significant difference between CBFt and CBFw $(p<0.0001$; Fig. 1B). The patients were divided into 2 groups according to CBFt: high CBF (CBFt > mean value; Fig. 2A) and low CBF (CBFt< mean value; Fig. 2B).

Clinical characteristics, maximum tumor diameters, suprasellar extension grades [12, 17], and incidence of postoperative hemorrhage are summarized in Table 1. In the high CBF group, there were 2 men and 5 women between 46 and 67 years of age (mean age: 50 years). In the low CBF group, there were 10 men and 11 women between 29 and 84 years of age (mean age: 59 years). Total tumor removal was achieved in 9 patients (43\%) in the low CBF group and 1 patient (14\%) in the high CBF group. There were no statistically significant differences between the 2 groups regarding the maximum tumor diameter, suprasellar extension grade, and extent of resection. Two of the 7 patients in the high CBF group suffered from visual acuity deterioration and visual field stenosis due to postoperative hemorrhage, while no patients in the low CBF group did $(p=0.014)$.

A representative case with high CBFt is shown in Fig. 3. A preoperative gadolinium-enhanced coronal T1weighted image (Fig. 3A) shows a pituitary adenoma with suprasellar extension (Grade C). The scaled color maps for CBV (Fig. 3C) and CBF (Fig. 3D) show greater CBV and CBF of the tumor (6.6 and 77.5, respectively) than of the normal-appearing white matter (1.9 and 18.4, respectively). A postoperative gadolinium-enhanced coronal T1-weighted image (Fig. 3B) shows expansion of the tumor due to postoperative hemorrhage.

\section{Discussion}

There are 4 grades of pituitary adenoma with suprasellar extension: 1) Grade A, 0- to 10-mm suprasellar extension occupying the suprasellar cistern; 2) Grade B, 10- to 20-mm suprasellar extension with elevation of the third ventricle; 3 ) Grade C, 20- to 30-mm suprasellar extension occupying the anterior third ventricle; and 4) Grade D, > 30-mm suprasellar extension beyond the foramen of Monro or a Grade C tumor with lateral extension [12]. There is a $39.5 \%$ risk of residual or recurrent tumors after an initial TSS on imaging for Grade $C$ and D adenomas [12]. Based on their prospective study, Honegger et al. reported that vertical intracranial extension was the strongest independent predictor of incomplete resection after TSS for pituitary adenomas with suprasellar extension [6]. A residual suprasellar tumor can cause postoperative hemorrhage, which can result in compression of the optic pathway and acute hydrocephalus $[13,20,21]$. Mortini et al. reported that complete giant pituitary adenoma removal was obtained in only 13 of 67 patients (19\%) after an initial TSS [13]. Swelling and bleeding of a residual suprasellar tumor or sellar hematoma occurred in 8 of 85 patients ( $9 \%$ treated by TSS. Saito et al. recommended that the intrasellar dead space and sellar floor not be reconstructed in patients undergoing subtotal or partial tumor removal during an initial TSS in an intentionally staged operation, allowing marked descent of the diaphragma sellae and suprasellar tumor, thus avoiding postoperative complications [17]. Zoda et al. reported that an open craniotomy might be selected as the initial operation in cases with the following factors: significant suprasellar extension, lateral extension, retrosellar 
extension, brain invasion with edema, firm tumor consistency, involvement or vasospasm of the arteries of the circle of Willis, and optic apparatus encasement or optic foramina invasion [21].

A combined simultaneous transsphenoidal and transcranial approach to large-to-giant pituitary adenomas has been adopted to maximize tumor excision and lower the risk of swelling and bleeding of the residual tumor $[1,3,9]$. Recently, Han et al. reported that an endoscopic endonasal TSS was their first choice for surgical management of giant pituitary adenomas with enlarged sellae turcicae [5]. They also prepared for a simultaneous open craniotomy approach if a tumor had 1 or more of the following characteristics: dumbbell shape; irregular shape with significant subfrontal, temporal, or intraventricular extension; previous surgical treatment; suspicious fibrous consistency; or encasement of the optic apparatus and/or cerebral arteries [5]. Nagata et al. introduced a fully endoscopic combined endonasalsupraorbital keyhole approach for complicated parasellar lesions. They demonstrated that the intraoperative endoscopic view from the supraorbital approach showed the suprasellar pituitary tumor became hyperemic and swollen, with progression of tumor debulking from the transsphenoidal approach [14]. Because neurosurgeons cannot be aware of this phenomenon when using the transsphenoidal route alone, the swelling tumor compresses the surrounding neurovascular structures, resulting in postoperative complications.

PCT has been reported to be useful in grading gliomas [2, 19], differentiating tumor prognosis from treatment-induced effects [7], and differentiating glioblastomas, lymphomas, and metastatic tumors [15]. In the present study, we demonstrated that pituitary adenomas had high CBVt and increased vascular density. Our results revealed that we could preoperatively identify patients with a high risk of postoperative hemorrhage from residual tumors based on high CBFt by PCT. Conversely, pituitary adenomas with low CBFt, even those with Grade $\mathrm{C}$ or $\mathrm{D}$ suprasellar extension, could be safely removed via TSS as an initial surgery.

Sakai et al. reported that arterial spin-labeled perfusion images from a 3T magnetic resonance scanner reflected the vascular density of nonfunctioning pituitary macroadenomas, which may be useful in the preoperative prediction of intra- and postoperative tumor hemorrhage [18]. PCT provides reliable information on tumor vasculature [2]. The linear relationship between attenuation changes on computed tomography and tissue concentration of contrast medium, as well as the lack of confounding sensitivity to flow artifacts, allow PCT to potentially offer a more accurate representation of tissue microvasculature than similar magnetic resonance perfusion studies [8]. Additionally, the use of PCT using a 320-row multidetector computed tomography system in the present study overcame the limitation of limited area of coverage by conventional PCT compared with magnetic resonance perfusion imaging.

The present study has limitations that are inherent to its retrospective design. In addition, our study included a relatively small number of cases of large and giant pituitary adenomas.

\section{Conclusions}


PCT may be useful in the preoperative prediction of postoperative residual tumor hemorrhage, which can lead to hazardous postoperative complications after TSS for large and giant pituitary adenomas. In cases of pituitary adenomas with large suprasellar extension and high CBFt, an open craniotomy might be considered in combination with TSS.

\section{Abbreviations}

$\mathrm{CBF}$

cerebral blood flow; CBV:cerebral blood volume; PA:pituitary adenoma; PCT:perfusion computed tomography; TSS:transsphenoidal surgery

\section{Declarations}

Ethics approval and consent to participate

Approval for this study was obtained from the institutional research ethics boardof Shizuoka Cancer Center (30-J93-30-1-3). Individual written informed consent was waived because this study was retrospective in design and based on clinical records.

Consent for publication

Not applicable.

Availability of data and material

The datasets analysed during the current study are available from the corresponding author on reasonable request.

Competing interest

The authors declare that they have no competing interests.

Funding

This work has not been funded.

Author Contributions

$\mathrm{NH}$ designed the study, and collected data with $\mathrm{AU}$. $\mathrm{NH}, \mathrm{KM}$, and SD participated in statistical analysis. $\mathrm{NH}$ and ME interpreted results, and prepared and drafted the manuscript. All authors read and approved the final manuscript.

Acknowledgements

Not applicable. 


\section{References}

1. Alleyne $\mathrm{CH}$, Barrow DL, Oyesiku NM. Combined transsphenoidal and pterional craniotomy approach to giant pituitary tumors. Sur Neurol. 2002;57:380-90.

2. Beppu T, Sasaki M, Kudo K, Kurose A, Takeda M, Kashimura H, kashamura H, Ogawa A, Ogasawara $\mathrm{K}$. Prediction of malignancy grading using Computed tomogramphy perfusion imaging in nonenhancing supratentorial gliomas. J Neurooncol. 2011;103:619-27.

3. D'Ambrosio AL, Syed ON, Grobelny BT, Freda PU, Wardlaw S, Bruce JN. (200) Pituitary 12:217 - 215.

4. Dehdashti AR, Ganna A, Karabatsou K, Gentili F. Pure endoscopic endonasal approach for pituitary adenoma: early surgicall results in 200 patients and compareson with previous microsurgical series. Neurosurgery. 2008;62:1006-15.

5. Han S, Gao W, Jing Z, Wang Y, Wu A. How to deal with giant pituitary adenomas: transphenoidal or transcranial, simultaneous or two-staged? J Neurooncol. 2017;132:313-21.

6. Honegger J, Ernemann U, Psaras T, Will B. Objective criteria for successful transsphenoidal removal of suprasellar nonfunctioning pituitary adenoma. A prospective study. Acta Neurochir (Wien). 2007;149:21-9.

7. Jain R, Scarpace L, Ellika S, Schltz LR, Rock JP, Rosenblum ML, Patel SC, Lee TY, Mikkelsen T. Firstpass perfusion computed tomography: Initial experience in differentiating recurrent brain tumors from radiation effects and radiation necrosis. Neurosurgery. 2007;61:778-87.

8. Jain R, Ellika SK, Scarpace L, Schltz LR, Rock JP, Gutierrez J, Patel SC, Ewing J, Mikkelsen T. Quantitative estimation of permeability surface-area product in astroglial brain tumors using perfusion CT and correlation with histopathologic grade. Am J Neuroradiol. 2008;29:694-700.

9. Leung GTK, Law HY, Hung KNH, Fan YW, Lui WM. Combined simultaneous transcranial and transsphenoidal resection of large-togiant pituitary adenomas. Acta Neurochir (Wien). 2011;153:1401-8.

10. Magro E, Graillon T, Lassave J, Casinetti F, Boissonneau S, Tabouret E, Fuentes S, Velly L, Gras R, Dufour $\mathrm{H}$. Complications related to the endoscopic endonasal transsphenoidal approach for nonfunctioning pituitary macroadenomas in 300 consecutive patients. World Neurosurg. 2016;89:442-53.

11. Mamelak AN, Carmichael J, Bonert VH, Cooper O, Melmed S. Single-surgeon fully endoscopic endonasal transphenoidal surgery: outcomes in three-hundred consecutive cases. Pituitary. 2013;16:393-401.

12. Mohr G, Hardy J, Comtois R, Beauregard H. Surgical management of giant pituitary adenomas. Can J Neurol Sci. 1990;17:62-6.

13. Mortini P, Barzaghi R, Losa M, Boari N, Giovanelli M. Surgical treatment of giant pituitary adenomas: strategies and results in a series of 95 consecutive patients. Neurosurgery. 2007;60:993-1004.

14. Nagata Y, Watanabe T, nagatani T, Takeuchi K, Chu J, Wakabayashi T. Fully endoscopic combined transsphenoidal and supraorbital keyhole approach for parasellar lesion. J Neurosurg. 
2018;128:685-94.

15. Onishi S, Kajiwara Y, Takayasu T, Kolakshyapati M, Ishifuro M, amitya VJ, Takeshima Y, Sugiyama K, Kurisu K, Yamasaki F. Perfusion computed tomogramphy parameters are useful for differentiating glioblastoma, jymphoma and metastasis. World Neurosurg. 2018;119:e890-7.

16. Powell M. Microscope transphenoidal surgery. Acta Neurochir (Wien). 2012;154:913-7.

17. Saito K, Kuwayama A, Yamamoto N, Sugita K. The transsphenoidal removal of nonfunctioning pituitary adenomas with suprasellar extension: The open sella method and intentionally staged operation. Neurosurgery. 1995;36:668-76.

18. Sakai N, Koizumi S, Yamashita S, Takehara Y, Sakahara H, Baba S, Oki Y, Hiramatsu H, namba H. Arterial spin-labeled perfusion imaging reflects vascular density in nonfunctioning pituitary macroadenoma. Am J Neuroradiol. 2013;34:2139-43.

19. Shankar JJ, Woulfe J, Silva VD, Nguyen TB. Evaluation of perfusion CT in grading and prognosticateon of high-grade gliomas at diagnosis: a pilot study. AJR Am J Roentgenol. 2013;200:W504-9.

20. Sinha S, Sharma BS. Giant pituitary adenomas - An enigma revisited. Microsurgical treatment strategies and outcome in a series of 20 patients. Br J Neurosurg. 2010;24:31-9.

21. Zada G, Du R, Laws ER Jr. Defining the "edge of the envelope": patient selection in treating complex sellar-based neoplasms via transsphenoidal versous open craniotomy. J Neurosurg. 2011;114:286300 .

\section{Tables}

\begin{tabular}{|l|r|r|r|r|}
\hline \multicolumn{1}{|l|}{ Table1. Clinical characteristics of 28 patients grouped according to tumor blood flow } & \\
\hline \hline Number & & High CBF & Low CBF & p \\
\hline Mean age & & 7 & 21 & \\
\hline Gender & Male & 50 & 59 & 0.23 \\
\hline & Female & 2 & 10 & 0.37 \\
\hline Fuctioning adenoma & & 5 & 11 & \\
\hline Mean muximum diameter $(m m)$ & & 2 & 3 & 0.41 \\
\hline Grade according to suprasellar extension & A & 30 & 30 & 0.95 \\
\hline & B & 1 & 5 & 0.57 \\
\hline & C & 3 & 9 & \\
\hline & D & 3 & 5 & \\
\hline Extent of resection & Total & 0 & 2 & \\
\hline & Subtotal & 1 & 9 & 0.09 \\
\hline Postoperative hemorrhage & Partial & 0 & 3 & \\
\hline & & 6 & 9 & \\
\hline & & 2 & 0 & 0.014 \\
\hline
\end{tabular}


Figures

a

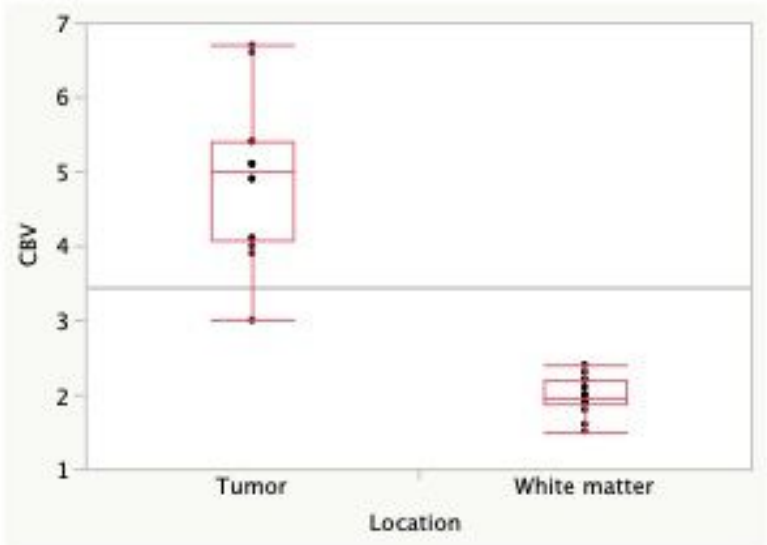

b

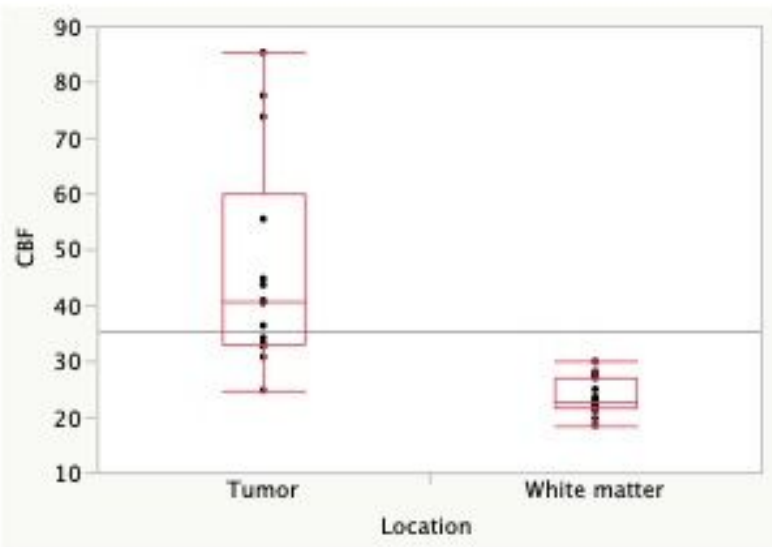

\section{Figure 1}

Box-and-whisker plots of CBV (a) and CBF (b) The horizontal bars inside of the boxes indicate medians. There were significant differences between both CBV and CBF of the tumor and white matter $(p<0.0001)$. 

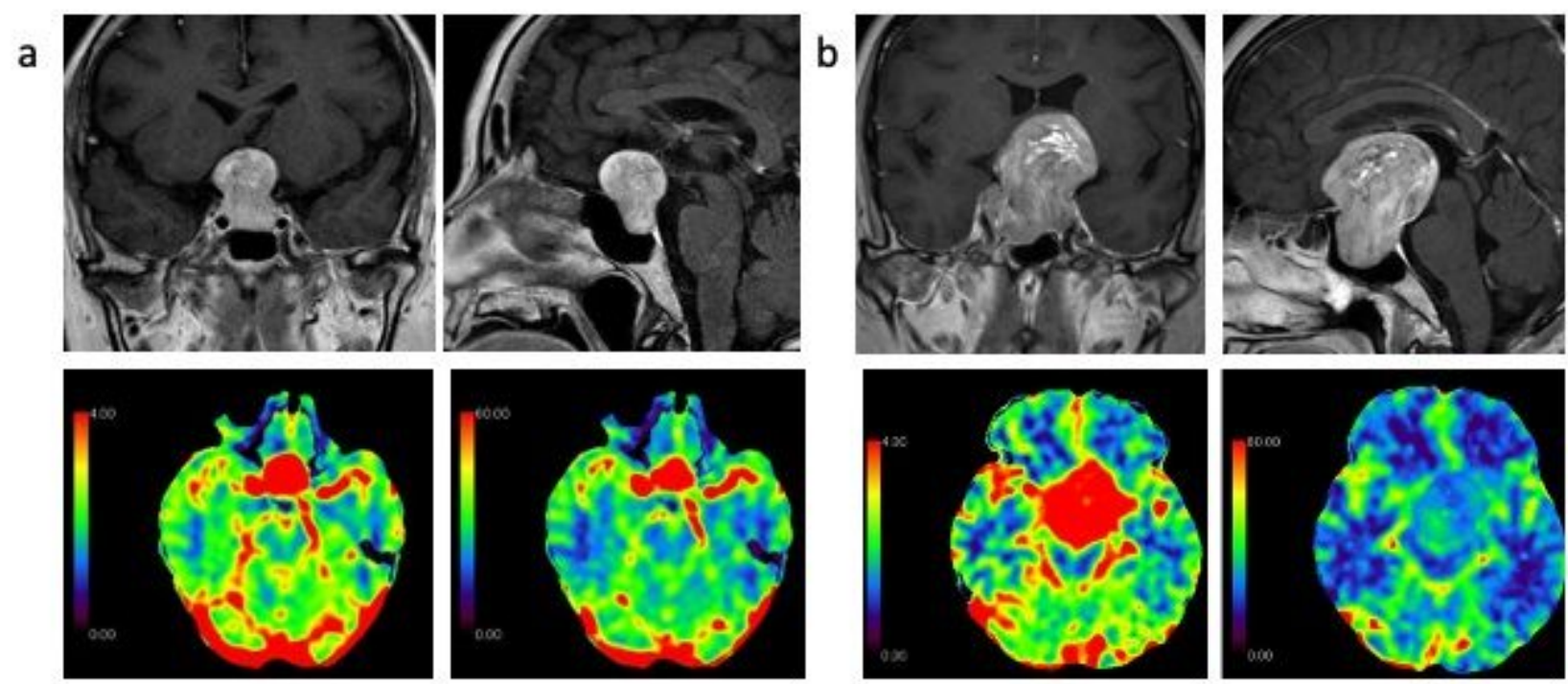

Figure 2

Representative images of patients in the high CBF (a) and low CBF (b) groups Preoperative gadoliniumenhanced coronal and sagittal T1-weighted images (upper panel, left and right, respectively) and scaled color maps for CBV (lower panel, left) and CBF (lower panel right). 

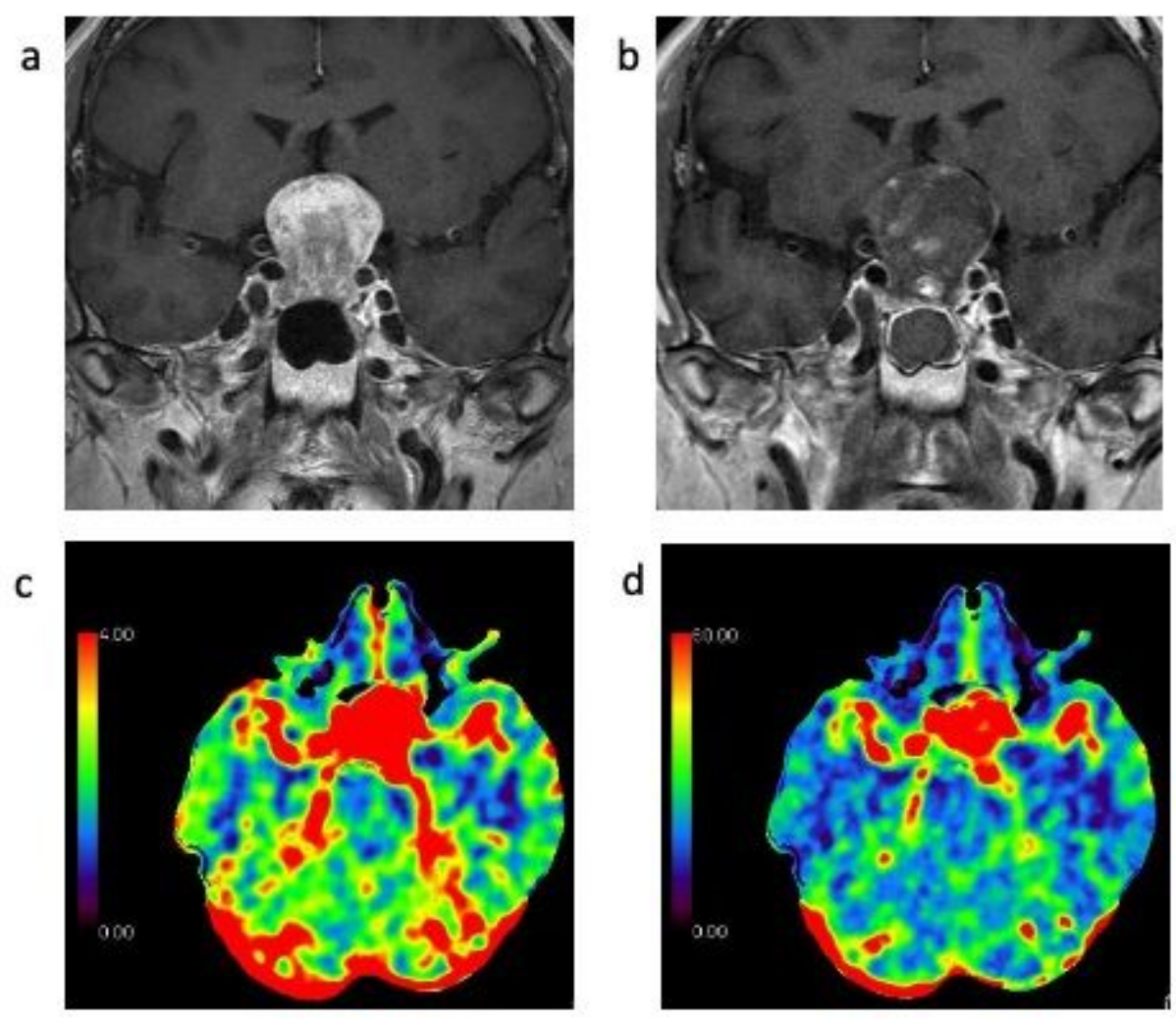

Figure 3

Preoperative (a) and postoperative (b) coronal gadolinium-enhanced T1-weighted images showing expansion of the tumor due to postoperative hemorrhage. Scaled color maps for CBV (c) and CBF (d) showing greater $\mathrm{CBV}$ and $\mathrm{CBF}$ of the tumor. 